\title{
Surface Forces and Structure in a Water-in-Salt Electrolyte
}

\author{
Timothy S. Groves, ${ }^{\dagger}$ Carla S. Perez-Martinez, ${ }^{\ddagger}$ Romain Lhermerout, ${ }^{\top}$ and Susan \\ Perkin ${ }^{*} \dagger$ \\ $\dagger$ Physical and Theoretical Chemistry Laboratory, University of Oxford, Oxford, UK \\ $\ddagger$ London Centre for Nanotechnology, University College London, London, UK \\ \Laboratoire Charles Coulomb, Université de Montpellier, CNRS, Montpellier, France \\ E-mail: susan.perkin@chem.ox.ac.uk
}

\begin{abstract}
Water-in-salt electrolytes are a fascinating new class of highly concentrated aqueous solutions with wide electrochemical stability windows that make them viable as aqueous battery electrolytes. However, the high ion concentration of water-in-salt electrolytes means that these systems are poorly understood when compared to more dilute electrolyte solutions. Here, we present direct surface force measurements across thin films of a water-in-salt electrolyte at several concentrations. We find that the electrolyte adopts a layered structure at charged interfaces composed of a nanostructure of hydrated cation and non-aqueous anion-rich domains. These observations will aid in the interpretation of capacitance and double layer behaviour of water-in-salt electrolytes with consequences for their use in energy storage devices.
\end{abstract}

\section{Keywords}

Water-in-Salt, Aqueous Electrolytes, High Concentration, Surface Forces, Nanostructure 
Electrolytes are ubiquitous: they form the planet's oceans, they exist within all living cells, and they are critical to the function of many modern technologies. In the dilute regime, electrolytes are well understood by considering the established ideal Debye-Hückel theory ${ }^{1}$ however as salt concentration increases and excluded volume and correlation effects become important, significant deviations from the theory are observed. ${ }^{2}$ Understanding the concentrated electrolyte regime is key in many areas, from the study of ion channels in biology ${ }^{3}$ to designing the next generation of electrochemical energy storage devices.

Water-in-Salt (WiS) electrolytes have recently emerged as promising candidates to replace conventional organic electrolytes in state of the art lithium ion batteries. ${ }^{4-7}$ These superconcentrated aqueous electrolytes, where the salt comprises the majority of the mass and volume of the system, are able to compete with traditional battery electrolytes owing to the vastly extended electrochemical stability windows of the aqueous system from $1.23 \mathrm{~V}$ in dilute electrolytes to $\approx 3 \mathrm{~V}$. This is possible as the high concentration of the WiS electrolyte allows for the efficient formation of a solid electrolyte interphase at the electrode surface that prevents breakdown of the electrolyte, as well as substantially reducing the activity coefficient of the water. ${ }^{4,6,8}$ Much work has been focused on attempting to understand these new electrolytes, in particular on modelling the environment of WiS electrolytes, ${ }^{9-11}$ on extending the WiS principle from lithium ion batteries to sodium, ${ }^{12,13}$ potassium ${ }^{14}$ and zinc ${ }^{15}$ ion batteries, and on broadening the electrochemical stability windows of WiS electrolytes. ${ }^{16,17}$ Despite this, the true nature of the structure of WiS electrolytes remains relatively poorly understood, as highlighted in a recent review article. ${ }^{18}$ In this communication, we present measurements of the surface forces and structure of aqueous lithium bis(trifluoromethylsulfonyl)imide (LiTFSI) in the concentrated regime in order to better understand the structure of WiS electrolytes in general and in particular at interfaces and in nanoconfinement.

Measurements were performed using a Surface Force Balance (SFB), shown schematically in Figure 1, previously described in detail ${ }^{22}$ and briefly summarised here. Full details of the SFB and the measurement process are given in the Supporting Information. Two 


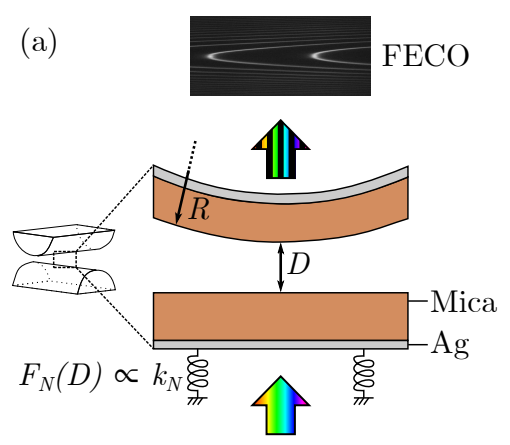

White Light

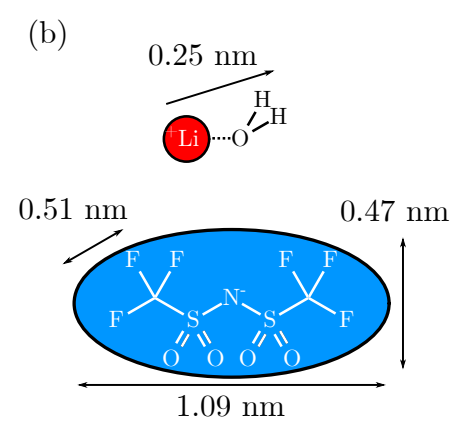

Figure 1: (a) Schematic of the SFB and interferometric cavity. (b) Approximate dimensions of the lithium-water primary hydration shell and TFSI anion, calculated from neutron scattering experiments ${ }^{19}$ and Van der Waals radii. ${ }^{20}$ (c) Phase diagram of the $\mathrm{H}_{2} \mathrm{O}$ - LiTFSI system adapted from a work by Ding et al. ${ }^{21}$ with the concentrations of the experiments detailed in this work shown by yellow circles. $\mathrm{I}=\mathrm{L}+\left(\mathrm{H}_{2} \mathrm{O}\right)_{4} \operatorname{LiTFSI}_{(\mathrm{s})}, \mathrm{II}=\mathrm{L}+\operatorname{LiTFSI}_{(\mathrm{s})}$, where $\mathrm{L}$ is a liquid solution.

back-silvered mica pieces of uniform thickness are glued, silver side down, to cylindrical glass lenses with radius $R \approx 10 \mathrm{~mm}$ using an epoxy resin (EPON 1004, Shell Chemicals). The lenses are mounted vertically in the instrument in a crossed cylinder configuration as shown in Figure 1(a), with the two silver mirrors forming an interferometric cavity. White light incident on the interferometer emerges as a series of Fringes of Equal Chromatic Order (FECO), analysis of which can determine the radius of the surfaces $R$ and the surface separation $D .{ }^{23}$ Distance dependent normal forces $F_{N}(D)$ are also determined from the FECO by measuring the deflection of a horizontal leaf spring upon which the lower lens is mounted. Calibrations for absolute distance measurements first take place in air, then a droplet of aqueous LiTFSI is confined between the mica surfaces.

The structure and dimensions of aqueous LiTFSI are shown in Figure 1(b). Solutions of aqueous LiTFSI are made up by dissolving LiTFSI from freshly opened bottles (Fluorochem, $99 \%)$ in ultrapure water $(18.2 \mathrm{M} \Omega \mathrm{cm}$, TOC $<2 \mathrm{ppb})$. The approximate concentrations investigated in this study are highlighted in the phase diagram in Figure 1(c) and detailed in table 1.

Measurements of equilibrium forces are made from an initial surface separation of $D \approx$ 
Table 1: Concentrations investigated in this study in molality, molarity, and water to salt ratio. $5 \mathrm{~m}$ represents the lower limit of the water-in-salt regime for this system. Densities are from a work by Han. ${ }^{24}$

\begin{tabular}{lll}
\hline Molality, $\mathrm{m}$ & Molarity, M & $\mathrm{H}_{2} \mathrm{O}: \mathrm{Li}$ \\
mol salt $/ \mathrm{kg} \mathrm{H} \mathrm{H}_{2} \mathrm{O}$ & mol salt / litre solution & molecular ratio \\
\hline 5 & 2.8 & $12: 1$ \\
8 & 3.8 & $7: 1$ \\
12 & 4.6 & $4.5: 1$ \\
\hline
\end{tabular}

$200 \mathrm{~nm}$. The surfaces are translated towards each other at constant velocity (in the range $0.5 \mathrm{~nm} \mathrm{~s}^{-1} \lesssim \mathrm{v} \lesssim 20 \mathrm{~nm} \mathrm{~s}^{-1}$ ) by means of either a mechanical or piezoelectric drive, while separation and force are measured directly. The resulting equilibrium forces are shown in Figure 2, normalised by the contact spot radius $R$ (measured in situ for each spot) to allow for quantitative comparison between different measurement spots, experiments and experimental geometries as described by the Derjaguin approximation, $F_{N}(D) / R=2 \pi W_{11}(D)$, where $W_{11}(D)$ is the interaction free energy between two parallel plates separated by a distance $D$.

At each concentration we observe a clear oscillatory force profile characterised by a series of repulsive walls extending to $\approx 7 \mathrm{~nm}$ surface separation. Retraction of the surfaces from each wall reveals an adhesive well. The force required to overcome each wall increases as surface separation decreases, similarly the force required to escape each well increases as the surface separation decreases. Forces can only be measured along the gradient of the spring constant $\left(\sim 120 \mathrm{Nm}^{-1}\right)$ hence regions of the force profile between the maxima of layer $i$ and the minimum of layer $i-1$ are inaccessible in our measurement and instead jumps are observed between repulsive walls and from adhesive wells.

These results are substantially different from those measured in simple dilute aqueous electrolytes, where the DLVO theory holds at the lowest concentrations measured with only small deviations close to contact as the concentration is increased due to the formation of a Stern layer of immobile ions at the surface. ${ }^{25,26}$ Instead, these results are more qualitatively similar i) to force profiles measured in concentrated solutions of aqueous simple salts 27,28 where oscillations are thought to arise from layering of hydrated cations neutralising the 

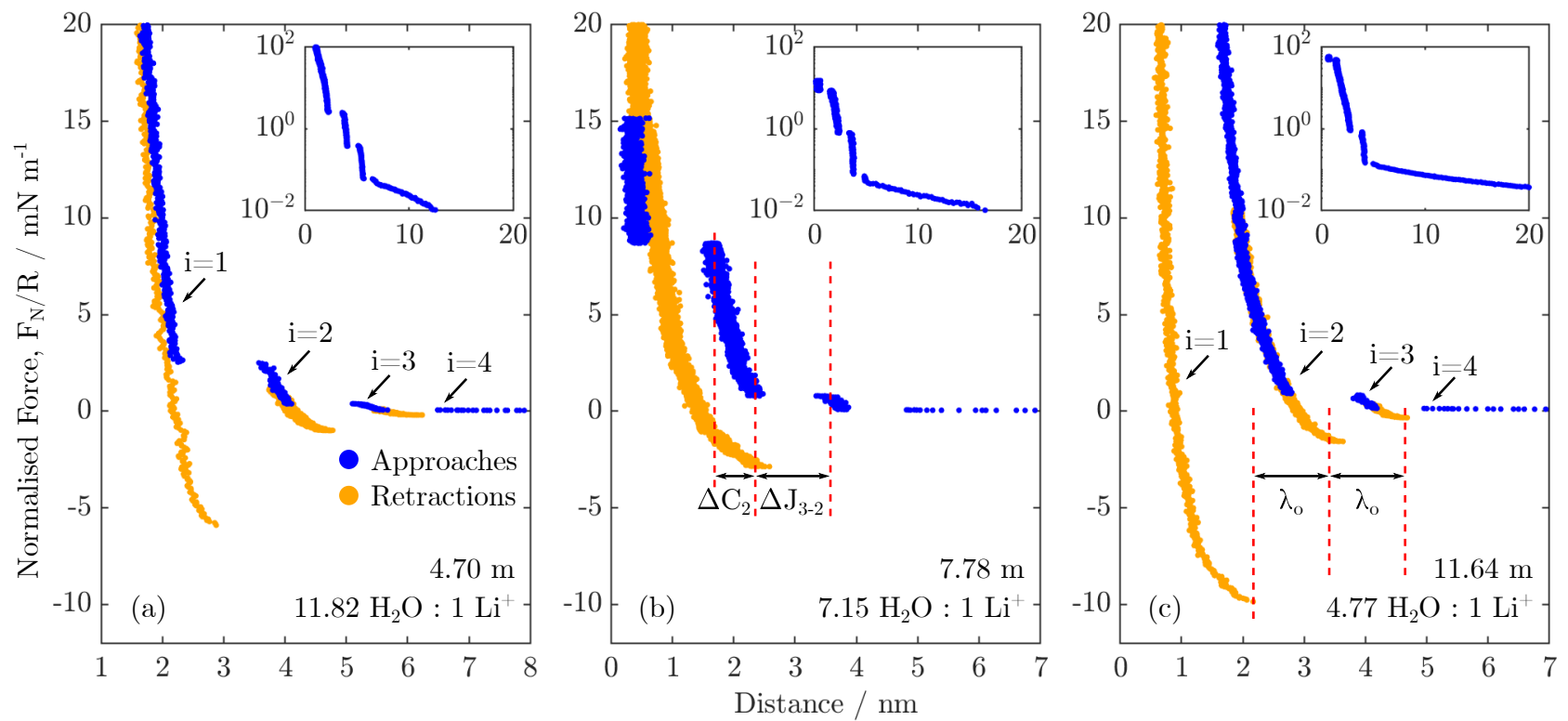

Figure 2: Force profiles measured at three concentrations of aqueous LiTFSI, normalised by the radius of the contact spot. (a) $4.70 \mathrm{~m}$. (b) $7.78 \mathrm{~m}$. (c) $11.64 \mathrm{~m}$. Forces measured on approach are shown in blue, and those on retraction in yellow. Layers are labelled from $i=1$ at closest approach as shown in (a) and (c), compressions $\Delta \mathrm{C}_{\mathrm{i}}$ within layers and jumps $\Delta \mathrm{J}_{\mathrm{i} \text {-(i-1) }}$ between layers are defined in the example in (b), while oscillatory wavelength $\lambda_{\mathrm{o}}$ is defined by linearly fitting minima positions and is shown in the example in (c) and in the Supporting Information. Small changes in the optical path can lead to an error in the absolute surface separation of up to $1 \mathrm{~nm}$, so runs have been shifted relative to the first approach on the first measurement spot in each case. Beyond the oscillatory force regime there is an exponentially decaying force, shown in the insets, with decay length $\lambda_{s}=8.3 \mathrm{~nm}$ at $7.78 \mathrm{~m}$ and $\lambda_{s}=11.5 \mathrm{~nm}$ at $11.64 \mathrm{~m}$. At $4.70 \mathrm{~m}$, this force is obscured by thermal drift. The greater line-width in panel (b) for $7.78 \mathrm{~m}$ is a result of thicker mica in that experiment. This can also introduce a small difference between $F(D) / R$ at the highest loads (smallest distances) when comparing between concentrations due to differences in contact mechanical stiffness. For this reason, we do not draw physical significance from the slight difference in closest separation between the $7.78 \mathrm{~m}$ and $11.64 \mathrm{~m}$ concentrations. 
surface charge; ii) to force profiles measured in ionic liquids (pure salts that are liquid at or near to room temperature), ${ }^{29,30}$ where oscillations are thought to arise from alternating layers of cations and anions and correspond to the ion pair diameter; and iii) to measurements in non-polar organic liquids, polymers and liquid crystals, ${ }^{31-33}$ where simple packing arguments are used to explain the layering and layer dimensions. We therefore suggest that the force profiles observed here arise from strong layering of species at the surfaces.

The dimensions and evolution with concentration of the layering wavelength, $\lambda_{o}$, jumps between layers, $\Delta \mathrm{J}_{\mathrm{i}-\mathrm{i}-1)}$, and compressions within layers, $\Delta \mathrm{C}_{\mathrm{i}}$, are shown in Figure 3. Interestingly, the values of $\lambda_{o}$ are consistently larger than can be accounted for by considering hydrated cation layers $(\approx 0.5 \mathrm{~nm}),{ }^{19}$ or by considering a simple ionic liquid-like ordered cation-anion layer set from the ion pair diameter $(\approx 0.7 \mathrm{~nm}) \cdot{ }^{30,34}$

The large layering dimensions suggest a self-assembled nanostructure within the liquid film. Such self assembled layers have previously been observed in SFB studies of long-chain ionic liquids ${ }^{20}$ where amphiphilic cations formed bilayers between the surfaces with layers composed of 'charge-rich' regions containing the anion and charged head group of the cation, and 'charge-poor' regions of interlocking non-polar cation tail groups.

Simplistically, lithium cations are small and charge dense, and are therefore well solvated in water, whereas TFSI anions are bulky and have delocalised charge centres, meaning they are less well solvated. We suggest that the liquid is nanostructured, consisting of a hydrated cation domain and a non-aqueous anion domain (Figure 4). The hydrated cation domain consists of lithium ions and water; the non-aqueous anion domain consists predominantly of anions with a small number of lithium ions, but with water molecules excluded. This arises because of the strongly hydrophobic nature of the anions in our electrolyte and the strongly hydrophilic lithium cations. This diverging affinity for water between cations and anions drives the charge separation in the nanostructure. These domains are templated against the SFB surfaces by the negative mica charge in a manner similar to that observed in ionic liquids. Each layer transition seen in Figure 2, with the wavelength of $\approx 1.4 \mathrm{~nm}$, 

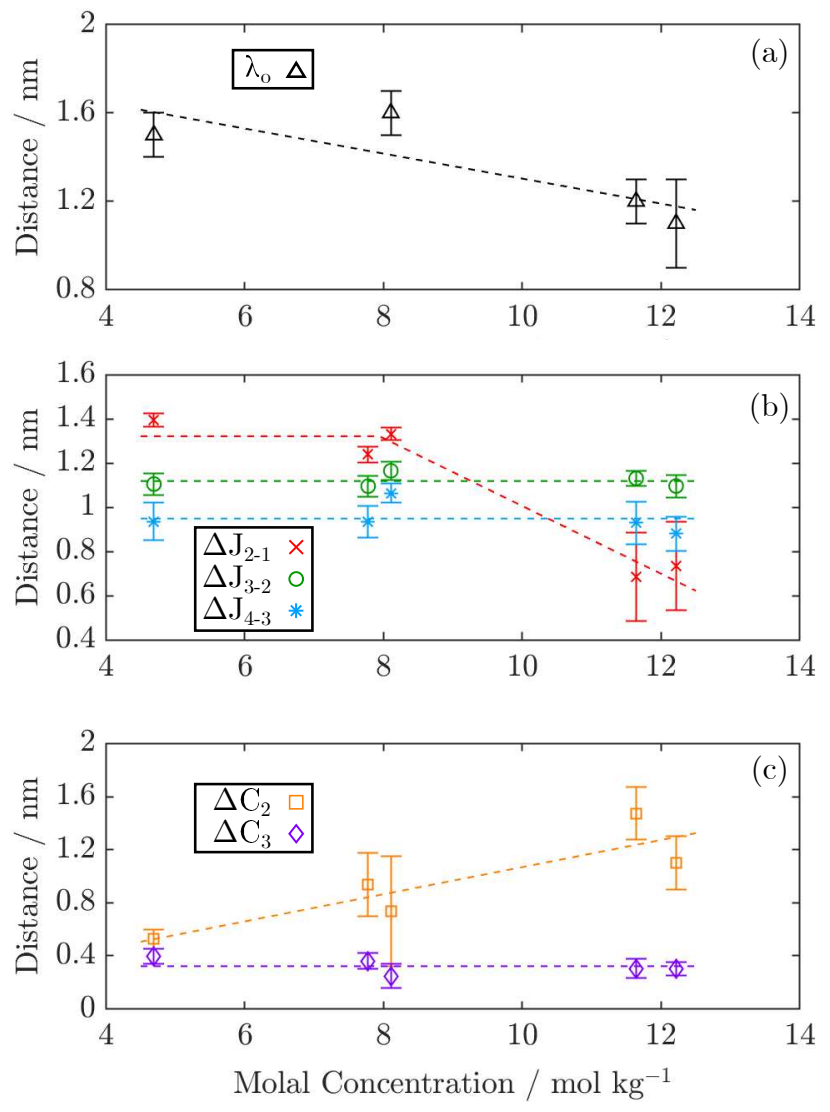

Figure 3: Layering data from aqueous LiTFSI. (a). Oscillatory wavelength $\lambda_{o}$ as a function of molal concentration, defined by a linear fit of jump out positions. (b). Jump distances $\Delta \mathrm{J}_{\mathrm{i}-(\mathrm{i}-1)}$ as a function of molal concentration of sample measured on approach. (c). Compressions $\Delta \mathrm{C}_{\mathrm{i}}$ within layers as a function of molal concentration as measured on approach. Examples of all quantities are shown in Figure 2. 
corresponds to the 'squeeze-out' of both a hydrated cation domain and a non-aqueous anion domain. This removal of both a cation domain and an anion domain from between the surfaces is driven by the requirement for charge neutrality between the SFB surfaces. This mechanism is analogous to the squeeze out of cations and anions as a single unit in SFB measurements of ionic liquids. ${ }^{20,30}$ A cartoon showing a portion of the WiS film is sketched in Figure 4 with the layering dimension highlighted.

Supporting this hypothesis, a similar scale of nanostructure has been observed previously in bulk LiTFSI. Borodin et al. performed a series of spectroscopic measurements and simulations at varying concentrations up to saturation of aqueous LiTFSI. ${ }^{35}$ They found evidence for a nanostructuring in the bulk liquid at high concentrations with a characteristic lengthscale of $1.4 \mathrm{~nm}$, similar to the measured oscillatory wavelengths observed here (Figure 3(a)). They attribute this to a 'solvent disproportionation', in which the liquid is separated on the nanoscale into a hydrated cation-rich $\mathrm{Li}^{+}\left(\mathrm{H}_{2} \mathrm{O}\right)_{4}$ domain, and a non-aqueous anion-rich $\mathrm{Li}^{+}\left(\mathrm{TFSI}^{-}\right)_{\mathrm{x}>1}$ domain. The SFB data presented here provides clear evidence for the existence of such a solvent-separated nanostructure at charged interfaces, which will have important consequences for the capacitance properties and behaviour of aqueous LiTFSI at electrode surfaces.

Recently, atomic force microscope (AFM) measurements were reported by Ichii et al. involving a similar WiS electrolyte containing a mixture of LiTFSI and lithium bis(pentafluoroethylsulfonyl)imide (LiBETI). In those experiments, layering was observed with smaller structural dimensions than in our present measurements. ${ }^{36}$ Potential causes for these differences may lie in the different WiS electrolyte composition studied, and the impact of the AFM tip geometry and chemistry. It is also important to note that the AFM experiments output a 'resonant frequency shift' of the AFM cantilever, compared to the direct surface normal force (and therefore interaction free energy) measured in SFB. The resonant frequency shift may detect sub-layers, collectively relating to the structural units observed in SFB. For example, the measurement with AFM of an outer layer of $\approx 0.9 \mathrm{~nm}$ followed by 
an inner layer layer of $\approx 0.6 \mathrm{~nm}$ could together correspond to our measurement of a single wavelength of $1.4 \mathrm{~nm}$ (precise matching is not expected as a result of the different molecular structures present). If this is the case, then together the AFM and SFB results complement each other and lead to a deeper understanding of the WiS structure.

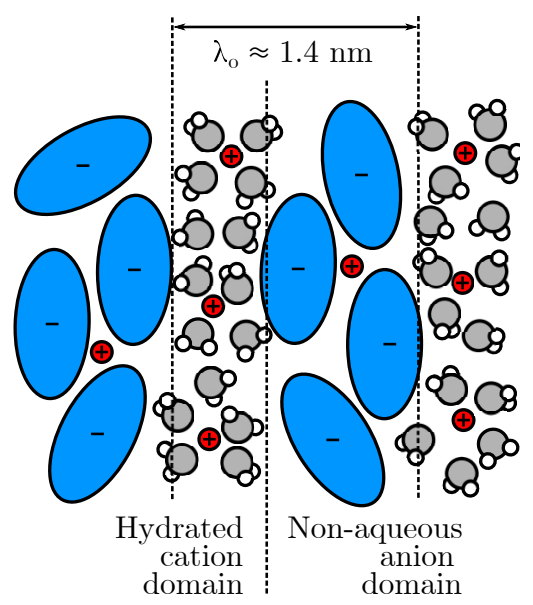

Figure 4: A cartoon representation of ion structure over two oscillatory wavelengths. Ions are separated into hydrated cation and non-aqueous anion rich domains. The oscillatory wavelength is the sum of the breadth ot the two domains.

Although $\lambda_{o}$ varies only by a small amount over the concentration range studied, the mechanism of the squeeze-out process appears to change more substantially. As concentration is increased, the force required to overcome the repulsive energy barrier and induce a layering transition also increases. Alongside this, compression within layer $i=2$ increases (Figure 3c). Whilst some part of this compression may arise within the mica layer, ${ }^{27,37}$ under the loads and mica thicknesses considered here, mica compression is unlikely to be responsible for the full effect observed. This suggests that under the high forces required to induce a layering transition, some reversible structural reorganisation can take place within the layers to reduce their thickness before the irreversible transition takes place. Such a reorganisation could occur via a dehydration within the hydrated cation domain, or by a a compression within the non-aqueous anion domain, resulting in a more compressed layer. The squeeze-out of this modified layer is responsible for the small jump to the final layer, $\Delta \mathrm{J}_{2-1}$, observed at the highest concentration investigated. 
The position at which the closest surface layer is first encountered varies with ion concentration. At $5 \mathrm{~m}$ the closest layer is observed at $2.1 \mathrm{~nm}$, however at $8 \mathrm{~m}$ and $12 \mathrm{~m}$ this decreases to $\approx 0.5 \mathrm{~nm}$. This variance likely corresponds to a changing mica surface charge with concentration. It is well known that potassium ions can be dissolved from mica sheets immersed in a dielectric liquid, leaving a negative charge on the surface. While the $\mathrm{K}^{+}$ions occur at such low concentration in the solution as to not affect the structure, the negative charge left on the surface can modify the ion ordering in the adjacent liquid. Potassium ion dissolution will happen to a greater extent at higher dielectric permittivities of solution, i.e. lower ion concentration, hence it is expected that the highest surface charge occurs at the lowest ion concentration studied. At the lowest concentration, the high surface charge means that the attraction between the surfaces and the adjacent layers is greater and therefore requires more force to overcome. The $2.1 \mathrm{~nm}$ distance observed is close to the dimensions of one oscillatory wavelength with a second hydrated cation domain, and we suggest that such a structure is held tightly enough to the surfaces to persist under the loads applied in this measurement. At the highest concentrations, the distance corresponds well to the diameter of a single hydrated cation domain that is able to neutralise the surface charges of both mica surfaces.

The insets of Figure 2 show the existence of a long range exponentially decaying monotonic force with an exponential decay length $\lambda_{s}$. This force is similar in appearance to the screening of charge within Debye-Hückel theory, however, the concentration regime in which we observe this screening is substantially higher than can be accounted for by considering a continuum theory approach. Instead we attribute this force to the recently observed underscreening phenomenon, which has been seen in ionic liquids ${ }^{28,38,39}$ and concentrated solutions of simple salts. ${ }^{28,40}$ The exponential decay length of the underscreening force increases with increasing concentration from $\lambda_{s}=8.3 \mathrm{~nm}$ at $7.78 \mathrm{~m}$ to $\lambda_{s}=11.5 \mathrm{~nm}$ at $11.64 \mathrm{~m}$ (at $5 \mathrm{~m}$ the decay length is obscured by thermal drift in the experiment). These values of $\lambda_{s}$ are of the same order as those observed previously in other concentrated electrolyte systems, and 
the observed increase of $\lambda_{s}$ with concentration is consistent with previous reports of under-

screening, ${ }^{28,40}$ however direct quantitative comparison of screening lengths is not meaningful until a theory incorporating strong assymetry of ion diameter emerges.

In summary, we have performed the first direct surface force measurements of aqueous solutions of the WiS electrolyte LiTFSI. We find that the system behaves differently to aqueous solutions of simple salts, showing well defined layers and long range screening forces similar to those observed in ionic liquids. They remain distinct from ionic liquids however, with surface forces dominated by a self-assembled nano-structure with a characteristic length scale $>1 \mathrm{~nm}$, suggesting the existence of large and separated cation and anion rich domains at charged surfaces. This result has interesting implications for the nature of WiS ion transport to the electrode, and will be important in future research to improve the efficacy of WiS electrolyte based electrochemical storage devices.

\section{Acknowledgement}

This research was funded by grants from The European Research Council (under Starting Grant No. 676861, LIQUISWITCH) and The Leverhulme Trust (RPG-2015-328).

\section{Supporting Information Available}

Additional details on the methods and data collection and analysis process

\section{References}

(1) Debye, P.; Huckel, E. Zur Theorie der Elektrolyte. I. Gefrierpunktserniedrigung und verwandte Erscheinungen. Physikalische Zeitschrift 1923, 24.

(2) Onsager, L. Theories of Concentrated Electrolytes. Chemical Reviews 1933, 13, 73-89. 
(3) Eisenberg, B. Interacting Ions in Biophysics: Real is not Ideal. Biophysical Journal 2013, 104, $1849-1866$.

(4) Suo, L.; Borodin, O.; Gao, T.; Olguin, M.; Ho, J.; Fan, X.; Luo, C.; Wang, C.; Xu, K. "Water-in-salt" electrolyte enables high-voltage aqueous lithium-ion chemistries. Science 2015, 350, 938-943.

(5) Suo, L.; Borodin, O.; Sun, W.; Fan, X.; Yang, C.; Wang, F.; Gao, T.; Ma, Z.; Schroeder, M.; von Cresce, A. et al. Advanced High-Voltage Aqueous Lithium-Ion Battery Enabled by "Water-in-Bisalt" Electrolyte. Angewandte Chemie International Edition 2016, 55, 7136-7141.

(6) Yamada, Y.; Usui, K.; Sodeyama, K.; Ko, S.; Tateyama, Y.; Yamada, A. Hydrate-melt electrolytes for high-energy-density aqueous batteries. Nature Energy 2016, 1, 16129.

(7) Suo, L.; Han, F.; Fan, X.; Liu, H.; Xu, K.; Wang, C. "Water-in-Salt" electrolytes enable green and safe Li-ion batteries for large scale electric energy storage applications. Journal of Materials Chemistry A 2016, 4, 6639-6644.

(8) Dubouis, N.; Lemaire, P.; Mirvaux, B.; Salager, E.; Deschamps, M.; Grimaud, A. The role of the hydrogen evolution reaction in the solid-electrolyte interphase formation mechanism for "Water-in-Salt" electrolytes. Energy Environ. Sci. 2018, 11, 3491-3499.

(9) Vatamanu, J.; Borodin, O. Ramifications of Water-in-Salt Interfacial Structure at Charged Electrodes for Electrolyte Electrochemical Stability. The Journal of Physical Chemistry Letters 2017, 8, 4362-4367.

(10) Li, Z.; Jeanmairet, G.; Méndez-Morales, T.; Rotenberg, B.; Salanne, M. Capacitive Performance of Water-in-Salt Electrolytes in Supercapacitors: A Simulation Study. The Journal of Physical Chemistry C 2018, 
(11) McEldrew, M.; Goodwin, Z. A. H.; Kornyshev, A. A.; Bazant, M. Z. Theory of the Double Layer in Water-in-Salt Electrolytes. The Journal of Physical Chemistry Letters 2018, 9, 5840-5846.

(12) Suo, L.; Borodin, O.; Wang, Y.; Rong, X.; Sun, W.; Fan, X.; Xu, S.; Schroeder, M. A.; Cresce, A. V.; Wang, F. et al. 'Water-in-Salt' Electrolyte Makes Aqueous Sodium-Ion Battery Safe, Green, and Long-Lasting. Advanced Energy Materials 2017, 7, 1701189.

(13) Kühnel, R.-S.; Reber, D.; Battaglia, C. A High-Voltage Aqueous Electrolyte for Sodium-Ion Batteries. ACS Energy Letters 2017, 2, 2005-2006.

(14) Leonard, D. P.; Wei, Z.; Chen, G.; Du, F.; Ji, X. Water-in-Salt Electrolyte for Potassium-Ion Batteries. ACS Energy Letters 2018, 3, 373-374.

(15) Hu, P.; Yan, M.; Zhu, T.; Wang, X.; Wei, X.; Li, J.; Zhou, L.; Li, Z.; Chen, L.; Mai, L. Zn/V2O5 Aqueous Hybrid-Ion Battery with High Voltage Platform and Long Cycle Life. ACS Applied Materials \&3 Interfaces 2017, 9, 42717-42722.

(16) Yang, C.; Chen, J.; Qing, T.; Fan, X.; Sun, W.; von Cresce, A.; Ding, M. S.; Borodin, O.; Vatamanu, J.; Schroeder, M. A. et al. 4.0 V Aqueous Li-Ion Batteries. Joule 2017, 1, $122-132$.

(17) Jiang, L.; Liu, L.; Yue, J.; Zhang, Q.; Zhou, A.; Borodin, O.; Suo, L.; Li, H.; Chen, L.; $\mathrm{Xu}, \mathrm{K}$. et al. High-Voltage Aqueous Na-Ion Battery Enabled by Inert-Cation-Assisted Water-in-Salt Electrolyte. Advanced Materials 2020, 32, 1904427.

(18) Chen, M.; Feng, G.; Qiao, R. Water-in-salt electrolytes: An interfacial perspective. Current Opinion in Colloid \& Interface Science 2020, 47, 99 - 110.

(19) Mason, P. E.; Ansell, S.; Neilson, G. W.; Rempe, S. B. Neutron Scattering Studies of the Hydration Structure of Li+. The Journal of Physical Chemistry B 2015, 119, 2003-2009. 
(20) Perkin, S.; Crowhurst, L.; Niedermeyer, H.; Welton, T.; Smith, A. M.; Gosvami, N. N. Self-assembly in the electrical double layer of ionic liquids. Chemical Communications 2011, 47, 6572-6574.

(21) Ding, M. S.; Xu, K. Phase Diagram, Conductivity, and Glass Transition of LiTFSI-H2O Binary Electrolytes. The Journal of Physical Chemistry C 2018, 122, 16624-16629.

(22) Klein, J.; Kumacheva, E. Simple liquids confined to molecularly thin layers. I. Confinement-induced liquid-to-solid phase transitions. The Journal of Chemical Physics 1998, 108, 6996-7009.

(23) Israelachvili, J. N. Thin film studies using multiple-beam interferometry. Journal of Colloid and Interface Science 1973, 44, 259-272.

(24) Han, S. Anionic effects on the structure and dynamics of water in superconcentrated aqueous electrolytes. $R S C A d v$. 2019, 9, 609-619.

(25) Israelachvili, J. N.; Adams, G. E. Measurement of forces between two mica surfaces in aqueous electrolyte solutions in the range 0-100 nm. J. Chem. Soc., Faraday Trans. 1 1978, 74, 975-1001.

(26) Pashley, R. Hydration forces between mica surfaces in aqueous electrolyte solutions. Journal of Colloid and Interface Science 1981, 80, 153 - 162.

(27) Espinosa-Marzal, R. M.; Drobek, T.; Balmer, T.; Heuberger, M. P. Hydrated-ion ordering in electrical double layers. Phys. Chem. Chem. Phys. 2012, 14, 6085-6093.

(28) Smith, A. M.; Lee, A. A.; Perkin, S. The Electrostatic Screening Length in Concentrated Electrolytes Increases with Concentration. Journal of Physical Chemistry Letters 2016, 7, 2157-2163.

(29) Horn, R. G.; Evans, D. F.; Ninham, B. W. Double-layer and solvation forces measured 
in a molten salt and its mixtures with water. The Journal of Physical Chemistry 1988, 92, 3531-3537.

(30) Perkin, S.; Albrecht, T.; Klein, J. Layering and shear properties of an ionic liquid, 1ethyl-3-methylimidazolium ethylsulfate, confined to nano-films between mica surfaces. Phys. Chem. Chem. Phys. 2010, 12, 1243-1247.

(31) Horn, R. G.; Israelachvili, J. N. Direct measurement of structural forces between two surfaces in a nonpolar liquid. The Journal of Chemical Physics 1981, 75, 1400-1411.

(32) Horn, R. G.; Israelachvili, J. N. Molecular organization and viscosity of a thin film of molten polymer between two surfaces as probed by force measurements. Macromolecules 1988, 21, 2836-2841.

(33) Horn, R. G.; Israelachvili, J. N.; Perez, E. Forces due to structure in a thin liquid crystal film. 1981, 42, 39-52.

(34) Atkin, R.; El Abedin, S. Z.; Hayes, R.; Gasparotto, L. H. S.; Borisenko, N.; Endres, F. AFM and STM Studies on the Surface Interaction of [BMP]TFSA and [EMIm]TFSA Ionic Liquids with Au(111). The Journal of Physical Chemistry C 2009, 113, 1326613272.

(35) Borodin, O.; Suo, L.; Gobet, M.; Ren, X.; Wang, F.; Faraone, A.; Peng, J.; Olguin, M.; Schroeder, M.; Ding, M. S. et al. Liquid Structure with Nano-Heterogeneity Promotes Cationic Transport in Concentrated Electrolytes. ACS Nano 2017, 11, 10462-10471.

(36) Ichii, T.; Ichikawa, S.; Yamada, Y.; Murata, M.; Utsunomiya, T.; Sugimura, H. Solvation structure on water-in-salt/mica interfaces and its molality dependence investigated by atomic force microscopy. Japanese Journal of Applied Physics 2020, 59, SN1003.

(37) Lhermerout, R.; Perkin, S. The influence of mechanical deformations on surface force measurements. 2020. 
(38) Gebbie, M. A.; Valtiner, M.; Banquy, X.; Fox, E. T.; Henderson, W. A.; Israelachvili, J. N. Ionic liquids behave as dilute electrolyte solutions. Proceedings of the National Academy of Sciences of the United States of America 2013, 110, 9674-9679.

(39) Gebbie, M. A.; Smith, A. M.; Dobbs, H. A.; Lee, A. A.; Warr, G. G.; Banquy, X.; Valtiner, M.; Rutland, M. W.; Israelachvili, J. N.; Perkin, S. et al. Long range electrostatic forces in ionic liquids. Chemical Communications 2017, 53, 1214-1224.

(40) Gaddam, P.; Ducker, W. Electrostatic Screening Length in Concentrated Salt Solutions. Langmuir 2019, 35, 5719-5727, PMID: 30945875. 


\section{Graphical TOC Entry}

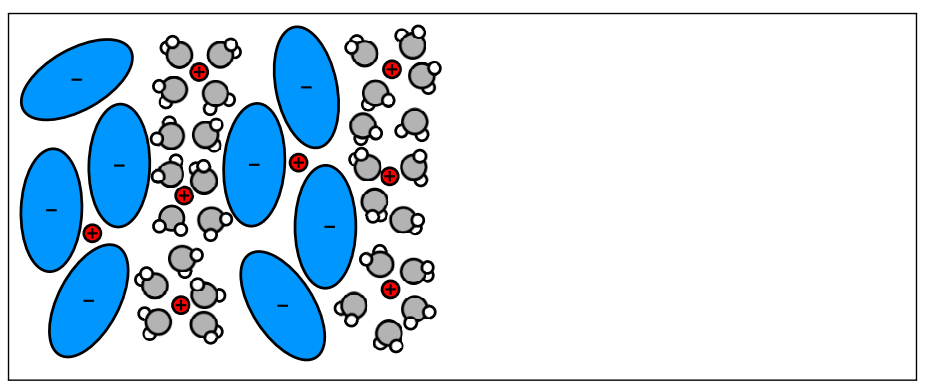

